\title{
An assessment of the functional outcomes of patients undergoing early exercise physiotherapy following arthroscopic rotator cuff repair using the double-row suture bridge technique
}

\author{
Nixon K. Dias*, Rakesh Sera
}

Department of Arthroscopy, Hosmat Hospital, Bangalore, Karnataka, India

Received: 04 September 2020

Revised: 21 October 2020

Accepted: 28 October 2020

*Correspondence:

Dr. Nixon K. Dias,

E-mail: nixonfiles@hotmail.com

Copyright: () the author(s), publisher and licensee Medip Academy. This is an open-access article distributed under the terms of the Creative Commons Attribution Non-Commercial License, which permits unrestricted non-commercial use, distribution, and reproduction in any medium, provided the original work is properly cited.

\begin{abstract}
Background: The methods of repairing rotator cuff tear are single-row technique, traditional double-row technique and suture-bridge technique. Studies have shown that the arthroscopic suture-bridge technique improved the pressurized contact area between the tendon and footprint. Controversy exists regarding the influence of early versus delayed motion on stiffness and healing rate after cuff repair. Early motion rehabilitation increases range of motion after cuff repair, although risk of re-tear is higher compared to immobilization. This study evaluated the functional outcomes in patients who underwent arthroscopic rotator cuff repair using the suture-bridge technique followed by an early exercise physiotherapy regime.

Methods: The study was a prospective and retrospective analysis of patients treated arthroscopically for rotator cuff tears in Hosmat hospital, Bangalore. 30 patients who had only a supraspinatus/infraspinatus tear were included in the study. The functional outcome was assessed by two scoring systems, UCLA and ASES scores. Post operatively, patients were immobilized for 3 weeks only. They were followed up at 3 weeks, 6 weeks, 12 weeks and at 6 months.

Results: Our study had 20 patients reporting a good outcome, whereas 9 patients had a fair outcome. Only 1 patient reported a poor outcome. The mean ASES scores improved from 20.43 to 77.87 whereas the UCLA score improved from 10.03 to 27.93 . None had re-tears.

Conclusions: The arthroscopic suture-bridge technique resulted in acceptable patient satisfaction and functional outcome as $67 \%$ of our patients had a "good" outcome. Early ROM exercise accelerated recovery from postoperative stiffness for patients after arthroscopic rotator cuff repair.
\end{abstract}

Keywords: Arthroscopic cuff, Early physiotherapy, Suture-bridge technique

\section{INTRODUCTION}

Rotator cuff disease encompasses a wide range of pathology from minimal bursal or articular side irritation and tendonitis to severe degenerative rotator cuff arthropathy. Epidemiological studies strongly support a relationship between age and cuff tears prevalence. Advances in procedure now allow arthroscopic repair of even the largest tears. Rotator cuff repair is one of the most frequent procedures performed in the shoulder joint. Surgical treatment of chronic rotator cuff tears is indicated when non operative treatment fails. The techniques of rotator cuff repair have evolved from traditional open repair, to arthroscopically assisted mini-open rotator cuff repair, to complete arthroscopic rotator cuff repair. Arthroscopic rotator cuff repair is now becoming the standard surgery for rotator cuff tear. 
Rotator cuff repairs seek to achieve adequate tendon fixation and to secure the fixation during the process of biological healing. All double-row repairs are technically more challenging, require additional anchors and are thus more expensive as well as more time-consuming procedures the methods of repairing rotator cuff tear under arthroscopy are single-row (SR) anchor fixing technique, traditional double-row (DR) anchor fixing technique and suture-bridge (SB) technique. The traditional DR technique has advantages over the SR fixation in the coverage and initial fixation strength of the rotator cuff. However, more anchors are used to reduce the healing area, and there are more knots on the surface of the rotator cuff, which are prone to form adhesions or cause new impingement. Biomechanical studies have shown that the recently introduced arthroscopic SB technique improved the pressurized contact area and mean pressure between the tendon and footprint compared with conventional DR techniques. In addition, SB repair may allow quick arthroscopic cuff repair with reduced knot impingement compared with conventional DR techniques. ${ }^{1}$

Due to the belief that the restoration of rotator cuff anatomic footprint can lead to superior healing rates and functional outcomes, Fealy et al defined double-row anchor repair with mini open incision for the first time in 2002. ${ }^{2}$ The development of arthroscopic double-row rotator cuff repair, which was defined by Lo et al in 2003, has opened a new era in the treatment of rotator cuff tears. ${ }^{3}$ These authors have proposed that with this technique, a wider restoration of the anatomic footprint could be achieved, the repair strength would be higher, there would be less stress on the anchors and knots and better healing rates could be achieved. However, since it is a more complicated and expensive surgery, questions regarding the cost effectiveness of DR repairs were raised after some studies found similar functional outcomes of SR and DR techniques. In 2006, Park et al have defined the arthroscopic Transosseous equivalent (TOE) technique, also known as the SB technique, based on the TOE, which had been used in open surgery as gold standard. ${ }^{4}$ With this technique, the authors aimed to achieve a better healing process by providing better tendon bone contact and less trauma to the torn tendon.

Good to excellent outcomes have been reported after rotator cuff repair in the short- and long-term follow-up in most cases. However, despite advances in instrumentation and surgical techniques, a risk of re-rupture of $20 \%$ to $60 \%$ has been observed. ${ }^{5}$ The TOE technique was designed to improve the biomechanical repair construct in an effort to improve some limitations of conventional SR and DR repairs. In the TOE repair, the suture limbs from the medial row of anchors are brought over the bursal side of the rotator cuff and secured to the lateral margin of the greater tuberosity footprint with a knotless anchor. In recent years, the SB method has become the mainstream method for performing arthroscopic rotator cuff repair (ARCR). Although several studies have reported excellent results of ARCR by the SB method, some authors have warned an associated risk of failure around the medial anchor, similar to that reported for the DR method. Cho et al reported failure of the medial rotator cuff when the medial row of mattress sutures passes through the rotator cuff in the SB method and discussed the possibility of strangulation and relatively quick necrosis of the rotator cuff tendon in the medial row. ${ }^{6}$ Therefore, although the mechanical characteristics achieved by the SB method are superior to those of other methods, it may not always be the best choice for full thickness rotator cuff tears.

Following ARCR, a period of movement restriction is advised; however, the optimal time of immobilization is unknown. It is common a practice to ask patients to use a sling for 6 weeks and avoid activities with the affected shoulder. This period is important to protect the tendon, allow healing, and prevent re-tear events. However, delayed motion may increase the risk of postoperative shoulder stiffness and muscle atrophy and delay functional recovery. ${ }^{7}$ In recent years, there is still controversy regarding the influence of early versus delayed motion on stiffness and healing rate after ARCR. It is known that early motion rehabilitation increases range of motion (ROM) after ARCR, but the risk of re-tear is significantly higher compared to immobilization. ${ }^{8}$

Exercise is generally recommended to restore motion and shoulder function after ARCR. From the perspective of rehabilitation, during the first 6 weeks after ARCR, early active exercises of the lower load should be prescribed to improve function and motor performance of the shoulder without overloading the early surgical repair.

This study was designed to evaluate the functional outcomes in patients who underwent arthroscopic rotator cuff repair using the SB technique followed by an early exercise physiotherapy regime.

\section{METHODS}

The study was a prospective and retrospective analysis of 30 patients treated arthroscopically for rotator cuff tears in Hosmat hospital, Bangalore from February 2020 to August 2020. The study included patients who have had at least 6 weeks of conservative management from onset of symptoms and at least 6 months follow up after operation. Patients who had only a supraspinatus/infraspinatus tear were included in the study. Also, patients who were diagnosed to have cuff tears on MRI and suspected to have cuff tears on clinical evaluation are included in the study. Patients who had less than 6 months follow up and those who had associated fractures with rotator cuff tears were excluded. All the patients fulfilling the above inclusion criteria were taken into study irrespective of age, sex and gender were included in the study. Other variables like type and size of tears, a smoking history and history of intra articular steroid injection was also analysed.

A proforma was designed which is to be filled by the patient himself/herself pre operatively and on his 
subsequent visits post operatively at 3 weeks, 6 weeks, 12 weeks and 6 months. The patient would fill the subjective data by themselves while the muscle strength and range of motion are assessed by the surgeon and documented. The functional outcome was assessed by two scoring systems, UCLA shoulder scoring and ASES shoulder score index.

All operations were performed in the beach-chair position with the patient under regional anaesthesia. For the SB technique, we used one or two Twinfix Ultra HA suture anchors, (5.5 mm, Smith and Nephew, US Professional) containing a suture eyelet and loaded with two no. 2 nonabsorbable braided sutures, placed just lateral to the articular surface of the humeral head. The sutures perforated the tendon in a horizontal mattress stitch configuration, with an identical procedure then applied for the second medial anchor. To establish the lateral row, suture bridge repair was achieved with one or two 4.5-mm knotless anchors (Swive Lock; Smith and Nephew) that were fully inserted perpendicular to the cortical surface of the humerus, distal to the medial anchor in conjunction with one suture from each medial anchor.

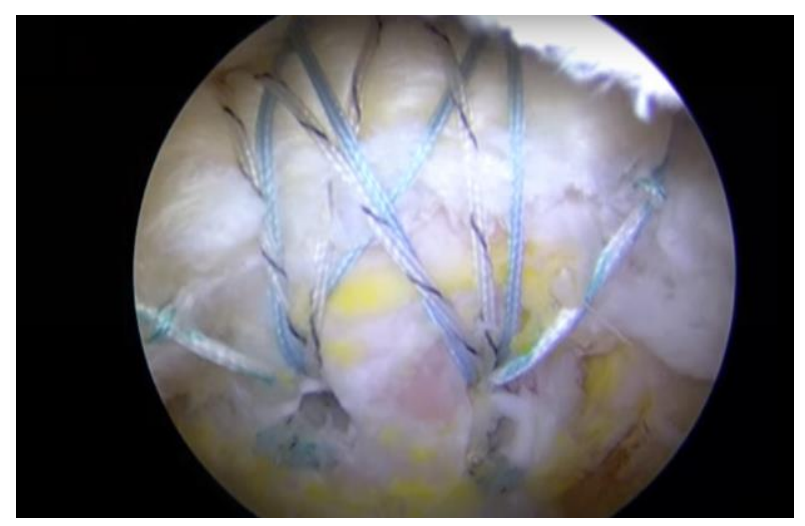

Figure 1: Completed arthroscopic rotator cuff repair with SB technique.

The same treatment regimen was prescribed to all patients, regardless of the repair status of the articular-side rotator cuff. Postoperatively, we recommended the use of a shoulder-immobilizing sling and provided instructions to maintain the shoulder at $30-40^{\circ}$ internal rotation. The patients performed gentle passive forward flexion exercises of the affected arm at the end of the second postoperative week. The sling was removed at 3 weeks postoperatively, and active mobilization was started. Active resistance-based muscle-strengthening exercises were started at 12 weeks postoperatively. At 3-4 months after surgery, the patients were permitted to perform light activities, with sports participation and heavy labor being allowed after 6 months. All subjects received a supervised early exercise program in combination with the standard exercise treatment.

The exercises should not have produced pain; only mild to moderate pain levels $(<4 / 10$ on the visual analog scale [VAS]) were accepted after the session. Each exercise and would be adjusted in relation to the individual patient. From 8 to 10 repetitions of each exercise would be performed, with 5 to 10 seconds of task maintenance and 30 seconds to 1 minute of rest between each repetition. There were weekly sessions for 6 weeks.

The data collected was entered into Microsoft excel spreadsheet and analyzed using IBM SPSS statistics, version 22 (Armonk, NY: IBM Corp). Descriptive data were presented in the form of frequency, percentage for categorical variables and in the form of mean, median, standard deviation and quartiles for continuous variables. Comparison of ASES between different time intervals was done using Wilcoxon signed rank. Comparison of UCLA between different time intervals was done using Friedman test. Comparison of change in UCLA and ASES between the age groups and tear size is done using ANOVA and Turkey Post Hoc test. Comparison of change in UCLA and ASES according to sex, tear type, etiology, smoking, IA steroid is done using independent sample $\mathrm{t}$ test. $\mathrm{P}$ value $<0.05$ was considered as statistically significant.

\section{RESULTS}

Most of the patients in our study belonged to the age group of 51-60 years. There was an uneven distribution among age groups, as seen in the graph.

The study had an equal proportion of males and females.

$27 \%$ of patients were found to have a partial thickness tear, whereas the remaining had a full thickness rotator cuff tear.

$20 \%$ of our study subjects had a tear due to a fall onto the shoulder, i.e. traumatic tear. Rest of the patients had degenerative tears, i.e. atraumatic tears.

$20 \%$ of the subjects had a smoking habit for the last 10 years at least.

When grouped according to the size of the cuff tears in subjects, $7 \%$ had a large tear, $36 \%$ had a medium sized tear whereas $57 \%$ of subjects had small tears.

$33 \%$ of the subjects had a history intra-articular steroid injection into the subacromial space before surgery.

Table 1: Distribution of study participants according to age.

\begin{tabular}{|cll|}
\hline Age (year) & Frequency & Percent $(\%)$ \\
\hline $\mathbf{2 1 - 3 0}$ & 4 & 13.3 \\
\hline $\mathbf{3 1 - 4 0}$ & 5 & 16.6 \\
\hline $\mathbf{5 1 - 6 0}$ & 16 & 53.3 \\
\hline $\mathbf{6 1 - 7 0}$ & 5 & 16.6 \\
\hline Total & 30 & 100.0 \\
\hline
\end{tabular}


Table 2: Comparison of ASES between baseline and 6 months.

\begin{tabular}{|lllllll|}
\hline ASES & N & Mean (SD) & Range & Median $(\mathbf{Q} 1-\mathbf{Q 3})$ & \multicolumn{2}{c}{ Wilcoxon signed ranks test } \\
\hline Baseline & 30 & $20.43(10.21)$ & $6.6-38.3$ & $23.20(9.20-28.30)$ & -4.79 & P value \\
\hline 6 months & 30 & $77.87(5.18)$ & $66.5-88.0$ & $80.00(74.50-81.50)$ & $-40.001 *$ \\
\hline
\end{tabular}

$* \mathrm{p}<0.05$ statistically significant, $\mathrm{p}>0.05$ non-significant, NS

Table 3: Comparison of UCLA between different time intervals.

\begin{tabular}{|c|c|c|c|c|c|c|}
\hline UCLA & $\mathbf{N}$ & Mean (SD) & Range & Median (Q1-Q3) & $\begin{array}{l}\text { Friedman test } \\
\text { Chi-square }\end{array}$ & P value \\
\hline Baseline & 30 & $10.03(2.59)$ & $6-15$ & $10(8-13)$ & \multirow{5}{*}{119.08} & \multirow{5}{*}{$<0.001$} \\
\hline 3 weeks & 30 & $12.17(2.26)$ & $8-17$ & $11(11-15)$ & & \\
\hline 6 weeks & 30 & $15.43(2.34)$ & $8-19$ & $16(13-17)$ & & \\
\hline 12 weeks & 30 & $19.17(3.03)$ & $14-24$ & $19(17-22)$ & & \\
\hline 6 months & 30 & $27.93(3.13)$ & $19-33$ & $29(27-30)$ & & \\
\hline
\end{tabular}

${ }^{*} \mathrm{p}<0.05$ statistically significant, $\mathrm{p}>0.05$ non-significant, NS

Table 4: Comparison of UCLA and ASES between the age groups.

\begin{tabular}{|c|c|c|c|c|c|c|c|c|}
\hline \multirow{2}{*}{ Variables } & \multirow{2}{*}{ Age (year) } & \multirow{2}{*}{$\mathbf{N}$} & \multirow{2}{*}{ Mean } & \multirow{2}{*}{ Std. Deviation } & \multirow{2}{*}{ Minimum } & \multirow{2}{*}{ Maximum } & \multicolumn{2}{|c|}{ ANOVA } \\
\hline & & & & & & & $\mathbf{F}$ & $P$ value \\
\hline \multirow{4}{*}{ UCLA } & 21-30 & 4 & 18.00 & 2.16 & 15 & 20 & \multirow{4}{*}{0.76} & \multirow{4}{*}{$0.53(\mathrm{NS})$} \\
\hline & $31-40$ & 5 & 18.80 & 4.60 & 11 & 23 & & \\
\hline & $51-60$ & 16 & 18.25 & 3.28 & 14 & 23 & & \\
\hline & 61-70 & 5 & 15.80 & 3.96 & 12 & 22 & & \\
\hline \multirow{4}{*}{ ASES } & 21-30 & 4 & 57.90 & 16.02 & 41.7 & 73.4 & \multirow{4}{*}{0.34} & \multirow{4}{*}{$0.80(\mathrm{NS})$} \\
\hline & $31-40$ & 5 & 57.74 & 10.76 & 46.7 & 73.4 & & \\
\hline & $51-60$ & 16 & 58.71 & 11.35 & 41.7 & 75.0 & & \\
\hline & $61-70$ & 5 & 52.70 & 9.77 & 36.7 & 63.4 & & \\
\hline
\end{tabular}

${ }^{*} \mathrm{p}<0.05$ statistically significant, $\mathrm{p}>0.05$ non-significant, NS

Table 5: Comparison of UCLA and ASES according to smoking.

\begin{tabular}{|c|c|c|c|c|c|c|c|c|c|c|}
\hline \multirow[t]{2}{*}{ Variables } & \multirow[t]{2}{*}{ Smoking } & \multirow[t]{2}{*}{$\mathbf{N}$} & \multirow[t]{2}{*}{ Mean } & \multirow[t]{2}{*}{ SD } & \multirow{2}{*}{$\begin{array}{l}\text { Mean } \\
\text { Difference }\end{array}$} & \multicolumn{2}{|c|}{$\begin{array}{l}95 \% \text { Confidence } \\
\text { Interval of Difference }\end{array}$} & \multirow[t]{2}{*}{$\mathbf{T}$} & \multirow[t]{2}{*}{ df } & \multirow{2}{*}{$\begin{array}{l}\mathbf{P} \\
\text { value }\end{array}$} \\
\hline & & & & & & Lower & Upper & & & \\
\hline \multirow{2}{*}{ UCLA } & Yes & 6 & 16.83 & 2.64 & \multirow{2}{*}{-1.33} & \multirow{2}{*}{-4.60} & \multirow{2}{*}{1.94} & \multirow{2}{*}{-0.84} & \multirow{2}{*}{28} & 0.41 \\
\hline & No & 24 & 18.17 & 3.66 & & & & & & (NS) \\
\hline ASES & Yes & 6 & 49.42 & 5.79 & -10.03 & -20.03 & -0.02 & -2.05 & 28 & $0.05^{*}$ \\
\hline
\end{tabular}

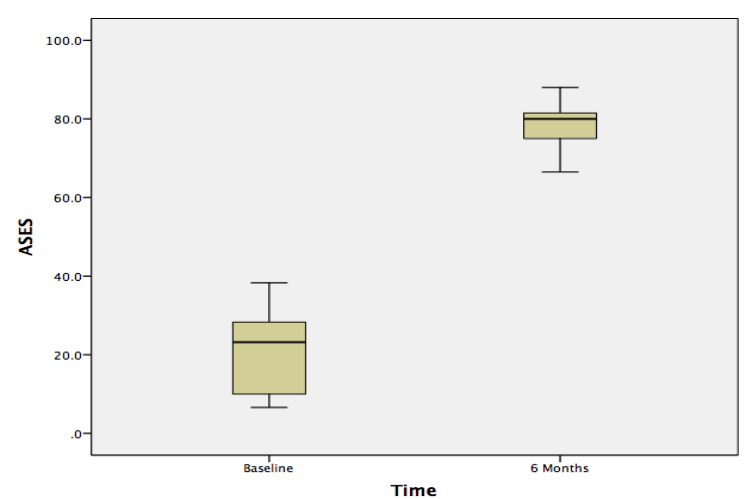

Figure 2: Comparison of ASES between baseline and 6 months.

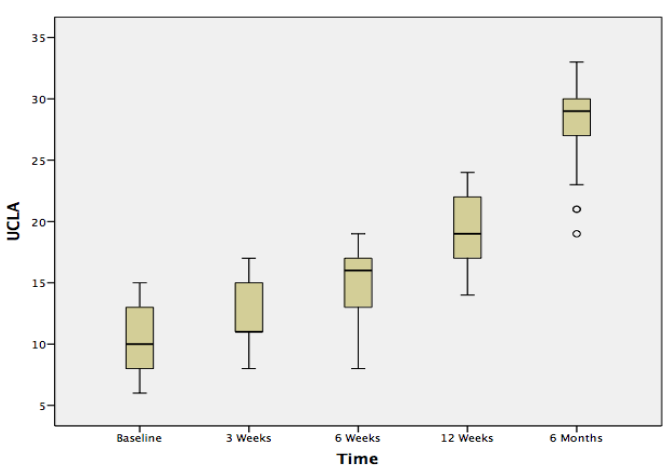

Figure 3: All pairwise comparison of UCLA between different time intervals using Wilcoxon signed ranks test is statistically significant $(\mathbf{p}<0.05)$. 
As seen above Figure 2 there was a significant improvement in ASES scores at the end of the 6 months follow-up.

The above Figures 3 show that there were significant improvements in UCLA scores among patients at each of the time intervals of follow up.

On assessing the results, in table 4 differences among outcomes were insignificant among various age groups.

\section{Independent sample t test}

The table 5 shows that, there was a significant decrease in the ASES scores among subjects with a smoking habit at the end of 6 months. On the other hand, presence of a smoking habit had no significant effect on the UCLA scores among subjects.

Table 6 (A): Comparison of UCLA and ASES according to tear size.

\begin{tabular}{|c|c|c|c|c|c|c|c|c|}
\hline \multirow{2}{*}{ Variables } & \multirow{2}{*}{ Tear size } & \multirow{2}{*}{$\mathbf{N}$} & \multirow{2}{*}{ Mean } & \multirow{2}{*}{$\begin{array}{l}\text { Std. } \\
\text { Deviation }\end{array}$} & \multirow{2}{*}{ Minimum } & \multirow{2}{*}{ Maximum } & \multicolumn{2}{|c|}{ ANOVA } \\
\hline & & & & & & & $\mathbf{F}$ & $P$ value \\
\hline \multirow{3}{*}{ UCLA } & Small & $\begin{array}{l}1 \\
7\end{array}$ & 18.71 & 3.33 & 13 & 23 & \multirow{3}{*}{3.37} & \multirow{3}{*}{$0.04 *$} \\
\hline & Medium & $\begin{array}{l}1 \\
1\end{array}$ & 17.64 & 3.14 & 12 & 23 & & \\
\hline & Large & 2 & 12.50 & 2.12 & 11 & 14 & & \\
\hline \multirow{3}{*}{ ASES } & Small & $\begin{array}{l}1 \\
7\end{array}$ & 60.35 & 11.43 & 36.7 & 75 & \multirow{3}{*}{1.98} & \multirow{3}{*}{0.16 (NS) } \\
\hline & Medium & $\begin{array}{l}1 \\
1\end{array}$ & 55.03 & 10.43 & 41.7 & 73.4 & & \\
\hline & Large & 2 & 45.95 & 6.01 & 41.7 & 50.2 & & \\
\hline
\end{tabular}

Table 6 (B): Pairwise comparison of UCLA according to tear size.

\begin{tabular}{|llllllll|}
$\begin{array}{l}\text { Dependent } \\
\text { Variable }\end{array}$ & $\begin{array}{l}\text { (I) Tear } \\
\text { size }\end{array}$ & $\begin{array}{l}\text { (J) Tear } \\
\text { size }\end{array}$ & $\begin{array}{l}\text { Mean } \\
\text { Difference } \\
\text { (I-J) }\end{array}$ & $\begin{array}{l}\text { Std. } \\
\text { Error }\end{array}$ & P value & $\begin{array}{l}\text { 95\% Confidence Interval } \\
\text { Lower } \\
\text { Bound }\end{array}$ & $\begin{array}{l}\text { Upper } \\
\text { Bound }\end{array}$ \\
\hline \multirow{2}{*}{ UCLA } & \multirow{2}{*}{ Small } & Medium & 1.07 & 1.25 & $0.67(\mathrm{NS})$ & -2.02 & 4.16 \\
\cline { 2 - 8 } & \multirow{2}{*}{ Medium } & Large & 6.21 & 2.41 & $0.04 *$ & 0.23 & 12.18 \\
& Large & 5.14 & 2.48 & $0.12(\mathrm{NS})$ & -1.01 & 11.28 \\
\hline
\end{tabular}

Tukey post Hoc test, ${ }^{*} \mathrm{p}<0.05$ statistically significant, $\mathrm{p}>0.05$ non-significant, NS

The table 6 (A) shows that, there was a significant decrease in the UCLA outcome scores depending on tear sizes. Larger tears had lower UCLA scores. On the contrary, there was no significant changes seen on comparison of the ASES scores.

As per these results in Table 6 (B), on pair wise comparison of the UCLA scores among different tear sizes, there was a significant decrease in subjects with large sized tears, as compared to those with small sized tears.

\section{DISCUSSION}

The goal of rotator cuff surgery is to alleviate shoulder pain and improve shoulder function. Arthroscopic rotator cuff repairs have been recently reported to show promising outcomes. The quality and function of rotator cuff muscles are known to deteriorate as age increases. In addition, the incidence of rotator cuff tear is known to increase with age, even in the asymptomatic population. Therefore, clarifying outcomes based on age is required for timing and prognosis of effective treatment.
The general observations in our study were as follows: Majority of patients were between age 51-60 years who had difficulty in carrying out their activities of daily living. There was an equal number of men and women in the study. Majority of patients the etiology was a degenerative tear, comprising $80 \%$. Majority of patients complained of inability to lift the shoulder following trauma. The incidence of partial thickness tears was 8 out of the 30 studied and full thickness tears were noted in the rest.

The correlation between patient age and outcome of rotator cuff repair was studied by Osti et al in 28 patients over 65 years of age and in 28 patients below 65 years. ${ }^{9}$ There was no statistical difference in functional outcome between the two groups. Verma et al studied arthroscopic rotator repair in 39 patients over 70 years of age. ${ }^{10}$ The pain score improved from $4.6 \pm 2.2$ to $0.5 \pm 0.9 \quad(\mathrm{p}<0.0001)$, and forward elevation increased from $114.8 \pm 42.0$ to $146.2 \pm 33.2^{\circ}(\mathrm{p}=0.0012)$. The results of the above studies are consistent with those of the current study. Our patients were divided into four age groups (21-30 years), (3-40 years), (51-60 years) and (61-70 years). At final follow-up, although the younger age group had better UCLA and ASES scores, as well as more strength at elevation and less pain with a higher satisfaction score, the difference was too 
small to be of statistical significance; probably due to the uneven distribution of subjects among age groups.

Recent arthroscopic repair techniques for rotator cuff tears have emphasized the potential for a double row repair to add strength to the repair and hopefully decrease the anatomic failure rate. Several studies have indicated that results in cases of anatomic failure, although clinically improved, are not as good as those that are anatomically intact, especially if strength measurements are made. Therefore, trying to achieve and maintain an intact cuff is a paramount goal in cuff repair. Biomechanical studies have emphasized the potential improvement of outcomes the TOE.

Park et al, in their study postulated that smoking affected healing failure after arthroscopic rotator cuff repair. ${ }^{4}$ In a study by Naimark et al, they studied the impact of smoking on patient outcomes after ARCR. They concluded, the minimal clinically important difference was achieved for ASES, WORC and VAS pain in both smokers and nonsmokers, suggesting both groups substantially benefit from arthroscopic rotator cuff repair. Smokers tend to have a lower functional improvement in response to surgery. ${ }^{11}$ Our study similarly showed that the functional outcomes were significantly lower in those with a smoking habit. Pain levels were also noted to be higher in the early follow up periods up to 3 weeks.

As a study by Liu et al quoted, the size of the tear at the time of the repair is a major determinant of the integrity of the cuff after repair. The integrity of the cuff at follow-up does not determine the functional outcome of the operated shoulder. Kim et al reported that arthroscopic repairs of medium and large full thickness tears of rotator cuffs had equal outcomes to technically unsuccessful arthroscopic repairs that were converted to mini-open repair techniques. ${ }^{13}$ Preoperative tear size is one of the major determinants of the outcome of rotator cuff repair. In our study, tear size determined intraoperatively was directly linked to lower functional outcomes as the tear sizes increased. There were significantly lower scores among patients with large tears (Cofield classification) as compared to those with small tears.

As per the results of a study done by Liu et al 80 and $88 \%$ good/excellent functional results were achieved in patients with full-thickness defects and no defects, respectively, without significant differences. ${ }^{14}$ Our study had 20 patients (67\% of subjects) reporting a good outcome, whereas 9 patients $(30 \%)$ had a fair outcome. Only 1 patient (3\%) of the subjects reported a poor outcome.

Allowing healing of the repaired rotator cuff tendon while minimizing stiffness and muscle atrophy are the primary goals of postsurgical rehabilitation. Unfortunately, there is a paucity of high-level evidence in the literature to support or develop various postoperative rehabilitation protocols. Koo and Burkhart presented both protective and accelerated rehabilitation protocols utilized based on individual patient risk for developing postoperative stiffness. The protective protocol, in which muscle strengthening exercises are delayed, is applied after arthroscopic repair of rotator cuff tears greater than $5 \mathrm{~cm}$ or involving more than 2 tendons, poor tissue quality, or repairs with greater tension. Each of these protocols implement various treatment modalities at different times to maximize healing and minimize stiffness based on repair characteristics such as tissue quality and repair robustness.

Chan et al in their study, concluded that the current metaanalysis did not identify any significant differences in functional outcomes and relative risks of recurrent tears between delayed and early motion in patients undergoing arthroscopic rotator cuff repairs. A statistically significant difference in forward elevation range of motion was identified; however, this difference is likely clinically unimportant. ${ }^{16}$ A study by Chang et al reported that "Early ROM exercise accelerated recovery from postoperative stiffness for patients after arthroscopic rotator cuff repair but was likely to result in improper tendon healing in shoulders with large-sized tears. The choice of either protocol should be based on an accommodation of the risks of recurrent tears and postoperative shoulder stiffness." 17

Patients in our study reported satisfactory outcomes at the final follow up at 6 months. Pain scores (VAS) was around 1 on activity and 0 at rest. They had a mean forward flexion of 150 degrees, abduction of 175 degrees and external rotation of 50 degrees. The mean ASES scores improved from 20.43 to 77.87 whereas the UCLA score improved from 10.03 to 27.93. Results were comparable to those of previous studies and none of the subjects in our study had any signs of re-tears.

The arthroscopic conventional suture-bridge technique resulted in acceptable patient satisfaction and functional outcome as majority of our patients $(67 \%)$ had a "good" outcome. The early exercise physiotherapy program resulted in satisfactory range of motion of the shoulder and helped in the reduction of stiffness, without causing any re-tears. Outcome scores were reduced with patients with history of smoking, probably due to decreased micro vascularization of the healing tendon to bone. Larger tears can also lead to a delayed recovery as compared to small tears, implying the importance of restoration of cuff anatomy and prevention of later gap formation. We find that the double-row suture bridge technique is useful in this regard. The age of the patient did not significantly affect the outcome scores, hinting that older age is not a deterrent for arthroscopic cuff surgery. A longer duration of postoperative follow up would have been beneficial in assessing the shoulder function and re-tear rates of this technique.

\section{ACKNOWLEDGEMENTS}

Authors would like to thank to my beloved teachers Dr. Raghunagaraj and Dr. Thomas Chandy for their 
continuous support during my dissertation work and related research, for their patience, motivation, and immense knowledge in the field of arthroscopy. I am also grateful to all the patients who participated in the study.

Funding: No funding sources

Conflict of interest: None declared

Ethical approval: The study was approved by the institutional ethics committee

\section{REFERENCES}

1. Ren YM, Zhang HB, Duan YH, Sun YB, Yang T, Tian MQ. Comparison of arthroscopic suture-bridge technique and double-row technique for treating rotator cuff tears: A PRISMA metaanalysis. Medicine (Baltimore). 2019;98(20):e15640.

2. Fealy S, Kingham TP, Altchek DW. Mini-open rotator cuff repair using a two-row fixation technique: outcomes analysis in patients with small, moderate, and large rotator cuff tears. Arthroscopy. 2002; 18:665-70.

3. Lo IKY, Burkhart SS. Double-row arthroscopic rotator cuff repair: re-establishing the footprint of the rotator cuff. Arthroscopy. 2003;19:1035-42.

4. Park MC, Elattrache NS, Ahmad CS, Tibone JE. Transosseous-equivalent" rotator cuff repair technique. Arthroscopy. 2006;22:1360:1-5.

5. Haque A, Pal Singh H. Does structural integrity following rotator cuff repair affect functional outcomes and pain scores? A meta-analysis. Shoulder Elbow. 2018;10(3):163-9.

6. Cho NS, Lee BG, Rhee YG. Arthroscopic rotator cuff repair using a suture bridge technique: is the repair integrity actually maintained? Am J Sports Med. 2011;39(10):2108-16.

7. Vo A, Zhou H, Dumont G, Fogerty S, Rosso C, Li X. Physical therapy and rehabilitation after rotator cuff repair: a review of current concepts. Int J Phys Med Rehabil. 2013;1(142):2.

8. Kelly BT, Roskin LA, Kirkendall DT, Speer KP. Shoulder muscle activation during aquatic and dry land exercises in nonimpaired subjects. J Orthop Sports Phys Ther. 2000;30(4):204-10.
9. Osti L, Papalia R, Del Buono A, Denaro V, Maffulli N. Comparison of arthroscopic rotator cuff repair in healthy patients over and under 65 years of age. Knee Surg Sport Trauma. 2010;18:1700-6.

10. Verma NN, Bhatia S, Baker Iii CL, Cole BJ, Boniquit $\mathrm{N}$, Nicholson GP. Outcomes of arthroscopic rotator cuff repair in patients aged 70 years or older. Arthroscopy. 2010;26:1273-80.

11. Naimark M, Robbins CB, Gagnier JJ, Landfair G, Carpenter J, Bedi A, Miller B. Impact of smoking on patient outcomes after arthroscopic rotator cuff repair. BMJ Open Sport \& Exercise Medicine. 2018;4(1):e000416.

12. Liu SH, Baker CL. Arthroscopically assisted rotator cuff repair: Correlation of functional results with integrity of the cuff. Arthroscopy. 1994;10:54-60.

13. Kim DH, Elattrache NS, Tibone JE, Jun BJ, DeLaMora SN, Kvitne RS, Lee TQ. Biomechanical comparison of a single-row versus double-row suture anchor technique for rotator cuff repair. Am J sports med. 2006;34(3):407-14.

14. Liu SH, Baker CL. Arthroscopically assisted rotator cuff repair: correlation of functional results with integrity of the cuff. Arthroscopy. 1994;10(1):54-60.

15. Koo SS, Burkhart SS. Rehabilitation following arthroscopic rotator cuff repair. Clin Sports Med. 2010;29(2):203-11.

16. Chan K, MacDermid JC, Hoppe DJ, Ayeni OR, Bhandari M, Foote CJ et al. Delayed versus early motion after arthroscopic rotator cuff repair: a metaanalysis. J Shoulder Elbow Surg. 2014;23(11):16319.

17. Chang KV, Hung CY, Han DS, Chen WS, Wang TG, Chien KL. Early Versus Delayed Passive Range of Motion Exercise for Arthroscopic Rotator Cuff Repair: A Meta-analysis of Randomized Controlled Trials. Am J Sports Med. 2015;43(5):1265-73.

Cite this article as: Dias NK, Sera R. An assessment of the functional outcomes of patients undergoing early exercise physiotherapy following arthroscopic rotator cuff repair using the double-row suture bridge technique. Int J Res Orthop 2021;7:6672. 